Results Mean follow-up duration was 10.7 years. Cut-off levels of $\mathrm{Lp}$ (a) for tertiles were $10 \mathrm{mg} / \mathrm{dl}$ and $23 \mathrm{mg} / \mathrm{dl}$. Risks for all stroke were 1.34 (95\% CI 1.03 to 1.74 ) and 1.00 (95\% CI 0.77 to 1.31) in the lower and the higher $\operatorname{Lp}(\mathrm{a})$ group, respectively, with reference to the middle group after adjustment for age, smoking status, drinking status, systolic blood pressure, and body mass index. Risks for cerebral haemorrhage (lower tertile 2.25, 95\% CI 1.28 to 3.94 and higher tertile 0.93 , $95 \%$ CI 0.49 to 1.77 ), were similar to all stroke and no significant relationships were seen between $\mathrm{Lp}(\mathrm{a})$ and cerebral infarction (lower tertile $1.15,95 \%$ CI 0.83 to 1.60 and higher tertile 1.02 , $95 \%$ CI 0.74 to 1.41 ),or subarachnoid haemorrhage (lower tertile $1.04,95 \%$ CI 0.52 to 2.09 and higher tertile $0.96,95 \%$ CI 0.48 to 1.90 ).

Conclusion Lower $\operatorname{Lp}(\mathrm{a})$ was an independent risk factor for stroke, especially, for cerebral haemorrhage in the general population.

\section{P2-125 INCIDENCE OF TYPE 2 DIABETES BY HBA 1 C AND OGTT: THE ISFAHAN DIABETES PREVENTION STUDY}

doi:10.1136/jech.2011.142976i.60

M Janghorbani, ${ }^{*}$ M Amini. Isfahan University of Medical Sciences, Isfahan, Iran

Aims The aim of this study was to estimate the incidence of type 2 diabetes using newly proposed haemoglobin $\mathrm{A}_{1 \mathrm{C}}\left(\mathrm{HbA}_{1 \mathrm{c}}\right)$ and current oral glucose tolerance test (OGTT) definition in an Iranian non-diabetic population.

Methods A total of 923 non-diabetic first-degree relatives of patients with type 2 diabetes 20-70 years old in 2003-2005 were followed through 2009 for the occurrence of type 2 diabetes. At baseline and through follow-ups, participants underwent a standard $75 \mathrm{~g}$ 2-h OGTT and $\mathrm{HbA}_{1 \mathrm{c}}$ measurements. Prediction of progression to type 2 diabetes by OGTT-defined or $\mathrm{HbA}_{1 \mathrm{c}}$-defined was assessed with area under the receiver-operating characteristic curves based upon measurement of fasting plasma glucose, 2 -h post-load glucose values and $\mathrm{HbA}_{1 \mathrm{c}}$

Results The prevalence of type 2 diabetes was $9.2 \%$ (95\% CI 8.2 to 10.2 ) by OGTT-defined diabetes and $7.9 \%$ (95\% CI 6.9 to 9.0 ) by $\mathrm{HbA}_{1 \mathrm{c}} \geq 6.5$. The incidence of type 2 diabetes was $2.0 \%$ (95\% CI 1.6 to 2.4$)(1.8 \%$ men and $2.1 \%$ women) per year by the current OGTT definition, whereas the incidence rates were $1.7 \%$ (95\% CI 1.3 to 2.0 ) (1.6\% men and $1.7 \%$ women) per year by $\mathrm{HbA}_{1 \mathrm{c}} \geq 6.5 \%$. Of those diagnosed with type 2 diabetes by OGTT, $69.6 \%$ had $\mathrm{HbA}_{1 \mathrm{c}}<6.5 \%$ and therefore would not have been classified as having type 2 diabetes.

Conclusions The incidence and prevalence of diabetes using newly proposed $\mathrm{HbA}_{1 \mathrm{c}}$ threshold in this first-degree relatives of patients with type 2 diabetes was slightly lower than using current OGTT definition.

\section{P2-126 WEIGHT CHANGE AND BLOOD PRESSURE, LIPIDS AND GLYCAEMIC CONTROL AMONG PATIENTS WITH TYPE 2 DIABETES}

doi:10.1136/jech.2011.142976i.61

M Janghorbani, ${ }^{*}$ M Amini. Isfahan University of Medical Sciences, Isfahan, Iran

Objective Although weight loss in patients with type 2 diabetes is very important, the data on the effect of long-term weight change on blood pressure (BP), lipids and glycaemic control among patients with type 2 diabetes receiving routine care are limited. The aim of this study was to assess the long-term impact of weight change on $\mathrm{BP}$, plasma lipids and glycaemic control among patients with type 2 diabetes receiving routine care.
Methods During the mean (SD) follow-up period of 9.2 (3.4) (range 2-15) years, 7712 patients with type 2 diabetes have been examined to determine changes in weight, BP, plasma lipids and glycaemic control using a linear mixed effects model for repeated measures. The mean (SD) age of participants was 51.3 (10.5) years with a mean (SD) duration of diabetes of 6.3 (6.3) years at initial registration.

Results The change in fasting plasma glucose and glycosylated haemoglobin $\left(\mathrm{HbA}_{1 \mathrm{c}}\right)$ from baseline to last follow-up examination was significantly more favourable in those who gain weight during follow-up than those who lost weight or stable weight. Systolic and diastolic BP and lipids also raised significantly more in the group with weight gain.

Conclusions Although this population of Iranian type 2 diabetes had negligible weight change over mean 9.2 years. Weight gain in patients with type 2 diabetes was associated with increase in BP and plasma lipids, but improvement in glycaemic control.

\section{P2-127 A CROSS SECTIONAL STUDY ON SEXUAL PRACTICES AND KNOWLEDGE RELATED TO SEXUAL HEALTH OF YOUTH IN THE REMOTE TEA PLANTATION SECTOR, SRI LANKA}

doi:10.1136/jech.2011.142976i.62

${ }^{1} \mathrm{~N}$ Jayasekara, ${ }^{1} \mathrm{P}$ Weerakoon, ${ }^{2} \mathrm{~S}$ Beneragama. ${ }^{1}$ The University of Sydney, Sydney, New South Wales, Australia; ${ }^{2}$ National STD/AIDS control Programme, Colombo, Sri Lanka

Background Issues related to sexual health among remote estate youth has gained a high priority in Sri Lanka.

General objective Describe the knowledge and practices related to sexual health of the youth in the tea plantation sector and conduct a pilot study on the sero - prevalence of common sexually transmitted diseases.

Specific objectives Explore the sexual health knowledge, educational needs and risk taking behaviours in the selected sample.

Methodology A descriptive cross sectional survey using a self administered questionnaire and interviews of 400 remote tea estate workers, aged between 18 and 24 years was carried out.

Results The sample consisted of 188 males (47\%) and 212 females (53\%), with a mean age 20.23 years. A total of 362 (90.5\%) were able to read and write. Peers were their main source of knowledge (59\%) and most reliable person to discuss sexuality ( $55 \%$ with a $95 \%$ CI of $50 \%$ to $59 \%$ ). The mean age of sexual debut for males was 12.56 $(\mathrm{SD}=1.88)$ and for females it was 16.21 years $(\mathrm{SD}=1.5)$. Eighty six percent of males had homosexual experience and $63 \%$ had more than one same sex partner. Their knowledge on STDs, HIV and available services were very low. None of the participants were test positive for HIV, Hepatitis B and Syphilis.

Conclusion Sexual health services are not sufficient to meet the needs of youth in the plantations and available services are not being delivered appropriately. A comprehensive, integrated sexual health service is needed for the youth and adolescents in the estate sector.

\section{P2-128 DISTRIBUTION OF 10-YEAR AND LIFETIME PREDICTED RISK FOR CARDIOVASCULAR DISEASE IN THE INDIAN SENTINEL SURVEILLANCE STUDY POPULATION}

doi:10.1136/jech.2011.142976i.63

${ }^{1,2} \mathrm{P}$ Jeemon, ${ }^{*}{ }^{1,3} \mathrm{D}$ Prabhakaran, ${ }^{4} \mathrm{M}$ Huffman, ${ }^{1,3} \mathrm{~S}$ Goenka, ${ }^{5} \mathrm{~L}$ Ramakrishnan, ${ }^{6} \mathrm{~K}$ R Thankappan, ${ }^{7} \mathrm{~V}$ Mohan, ${ }^{8} \mathrm{P}$ P Joshi, ${ }^{4} \mathrm{D} \mathrm{M}$ Lloyd-Jones, ${ }^{3} \mathrm{~K}$ S Reddy. ${ }^{1}$ Centre for Chronic Disease Control, New Delhi, India; ${ }^{2}$ Institute of Cardiovascular and Medical Sciences, University of Glasgow, Glasgow, UK; ${ }^{3}$ Public Health Foundation of India, New Delhi, India; ${ }^{4}$ Feinberg School of Medicine, Northwestern University, Chicago, USA; ${ }^{5}$ All India Institute of Medical Sciences, New Delhi, India, New Delhi, India; ${ }^{6}$ Sree 
Chitra Tirunal Institute of Medical Sciences and Technology, Trivandrum, India; ${ }^{7}$ Madras Diabetes Research Foundation, Chennai, India; ${ }^{8}$ Indira Gandhi Government Medical College, Nagpur, India

Introduction Cardiovascular disease (CVD) prevention guidelines recommend lifetime risk stratification for primary prevention of CVD, but no such risk stratification has been performed in India to date.

Methods We estimated short-term and lifetime predicted CVD risk among 10054 disease free, adult Indians in the age group of 20-69 years who participated in a nationwide risk factor surveillance study. The study population was then stratified into high shortterm ( $\geq 10 \% 10$-year risk or diabetes), low short-term $(<10 \%) /$ high lifetime and low short-term/low lifetime CVD risk groups.

Results The mean age (SD) of the study population (men=63\%) was $40.8+10.9$ years. High short-term risk for coronary heart disease was prevalent in more than one fifth of the population $(23.5 \%, 95 \%$ CI 22.7 to 24.4). Nearly half of individuals with low short-term predicted risk $(48.2 \%, 95 \%$ CI 47.1 to 49.3$)$ had a high predicted lifetime risk for CVD. While the proportion of individuals with all optimal risk factors was $15.3 \%$ (95\% CI 14.6 to 16.0 ), it was $20.6 \%$ (95\% CI 18.7 to 22.6 ) and $8.8 \%$ (95\% CI 7.7 to 10.5$)$ in the highest and lowest educational groups, respectively.

Conclusion Approximately 1 in 2 men and 3 in 4 women in India had low short-term predicted risks for CVD in this national study, based on aggregate risk factor burden. However, 2 in 3 men and 1 in 2 women had high lifetime predicted risks for CVD, highlighting a key limitation of short-term risk stratification.

\section{P2-129 PRE-PREGNANCY BODY MASS INDEX AND BLOOD PRESSURE IN THE FIRST TRIMESTER OF PREGNANCY: PRELIMINARY RESULTS FROM A COHORT OF RIO DE JANEIRO, BRAZIL}

\section{doi:10.1136/jech.2011.142976i.64}

F Rebelo, A B Franco-Sena, D R Farias, J dos Santos Vaz, G Kac.* Rio de Janeiro Federal University, Rio de Janeiro, Brazil

Background Women who have higher systolic blood pressure (SBP) and diastolic blood pressure (DBP) in early pregnancy are more prone to develop pregnancy complications such as hypertension, preeclampsia and eclampsia. These complications contribute significantly to morbidity, stillbirth and neonatal mortality.

Objective To evaluate the effect of pre-pregnancy body mass index (BMI) on first trimester SBP, DBP and mean arterial pressure (MAP). Methods 118 pregnant women between 20 and 40 years of age, up to 13 weeks and free from chronic or infectious diseases were investigated. SBP and DBP were obtained with automatic arm sphygmomanometer (Omron) and MAP was calculated as (SBP $+\mathrm{DBP} \times 2) / 3$. Statistical analysis was performed using ANOVA, correlation and multivariate linear regression. Adjustments were made for age, skin colour, parity and smoking.

Results Women were 26.3 44.9 years of age, had $8.7 \pm 2.7$ years of schooling, per-capita income (US\$) of $291.2 \pm 166.4$ and pre-pregnancy BMI of $25.6 \pm 5.1 \mathrm{~kg} / \mathrm{m}^{2}$. There was a moderate positive correlation between pre-pregnancy BMI and SBP $(r=0.52 ; p<0.001)$, $\operatorname{DBP}(r=0.33 ; \mathrm{p}<0.001)$ and MAP $(\mathrm{r}=0.44 ; \mathrm{p}<0.001)$. Mean MAP $(\mathrm{mm} \mathrm{Hg})$ increased with BMI categories (underweight $=76.5$; normal weight $=77.4$; overweight $=82.9$ and obesity $=88.7, p<0.001$ ) as did SBP (underweight $=100.3$; normal weight $=105.8$; overweight $=112.4$ and obesity $=121.2, \quad \mathrm{p}<0.001$ ) and DBP (underweight $=64.8 ;$ normal weight $=63.5 ; \quad$ overweight $=68.3$ and obesity $=72.6, \mathrm{p}<0.001)$. Multivariate linear regression showed that an increase of $1.0 \mathrm{~kg} / \mathrm{m}^{2}$ in pre-pregnancy BMI raised SBP in $1.24 \mathrm{~mm} \mathrm{Hg}(\mathrm{p}<0.001)$ and $0.53 \mathrm{~mm} \mathrm{Hg}$ in DBP $(p=0.002)$.
Conclusion Women who begin pregnancy overweight or obese tend to have higher values of SBP and DBP, which may indicate a higher risk of developing hypertensive disorders during pregnancy.

\section{P2-130 EPIDEMIOLOGY OF HYPERTENSION IN AN URBAN SRI LANKAN POPULATION}

doi:10.1136/jech.2011.142976i.65

${ }^{1} \mathrm{~A}$ Kasturiratne, ${ }^{*}{ }^{1} \mathrm{~T}$ Warnakulasuriya, ${ }^{1} \mathrm{~J}$ Pinidiyapathirage, ${ }^{2} \mathrm{~N}$ Kato, ${ }^{1} \mathrm{R}$ Wickremasinghe, ${ }^{1} \mathrm{~A}$ Pathmeswaran. ${ }^{1}$ Department of Public Health, Faculty of Medicine, University of Kelaniya, Ragama, Sri Lanka; ${ }^{2}$ Department of Gene Diagnostics and Therapeutics, Faculty of Medicine, University of Kelaniya, Tokyo, Japan

Introduction Hypertension is a common risk factor for cardiovascular disease. In Sri Lanka, despite the existence of a universal free health system, services are not available for routine screening of hypertension in the general population. This paper aims to describe the epidemiology of hypertension in 35-64 year old residents in Ragama Medical Officer of Health area in the Gampaha district, Sri Lanka.

Methods An age-stratified random sample of 4400 adults between 35 and 64 years of age drawn from the population based electoral list, was invited for a screening programme on cardiovascular risk factors. Socio-demographic and risk factor related data and anthropometric and blood pressure measurements were obtained by trained research assistants. Blood was obtained for relevant biochemical investigations.

Results The prevalence of hypertension (systolic $>139 \mathrm{~mm} \mathrm{Hg}$ and/or diastolic $>89 \mathrm{~mm} \mathrm{Hg}$ ) in 2986 subjects (Males 45\%), was $30.4 \%$ (27.8\% in males; $32.5 \%$ in females). $31.8 \%(n=288)$ were previously undetected. Of the known hypertensives, $19.5 \%$ were not on anti-hypertensive medication and only $32.1 \%$ were controlled (defined by systolic <140 $\mathrm{mm} \mathrm{Hg}$ and diastolic <90 mm Hg). Factors associated with hypertension in both males and females were body mass index, waist circumference, fasting blood glucose and serum triglycerides.

Conclusions The prevalence observed is comparable to the prevalences of developed countries with relatively older populations. A considerable proportion of known hypertensives are not on treatment and the observed poor control indicates problems in drug compliance. Interventions targeting lifestyle modification and drug compliance are essential to control adverse outcomes of hypertension.

\section{P2-131 FALLING PREVALENCE OF IMPAIRED GLUCOSE TOLERANCE IN SOUTH ASIAN POPULATIONS}

doi:10.1136/jech.2011.142976i.66

${ }^{1} \mathrm{~S}$ V Katikireddi, ${ }^{2} \mathrm{~J}$ Morling, ${ }^{2} \mathrm{R}$ Bhopal. ${ }^{1} \mathrm{NHS}$ Lothian, Edinburgh, Scotland, UK ${ }^{2}$ Centre for Population Health Sciences, University of Edinburgh, Edinburgh, Scotland, UK

Introduction Recently, diabetes prevalence has soared in South Asians making it a global public health priority. However, there are suggestions from the PODOSA trial and elsewhere that pre-diabetes, including impaired glucose tolerance (IGT), may not be increasing. We conducted a systematic review to assess secular trends in prediabetes in South Asian populations worldwide.

Methods We searched electronic databases from inception to June 2009 for cross-sectional studies providing prevalence of pre-diabetes (using WHO criteria) in South Asian adult populations. Two reviewers independently screened articles, performed data extraction, quality appraisal and study classification with any discrepancies resolved by consensus. Repeated cross-sectional studies, 